\title{
On Angles Whose Squared Trigonometric Functions Are Rational*
}

\author{
J. H. Conway, ${ }^{1}$ C. Radin, ${ }^{2}$ and L. Sadun ${ }^{2}$ \\ ${ }^{1}$ Department of Mathematics, Princeton University, \\ Princeton, NJ 08544, USA \\ ${ }^{2}$ Department of Mathematics, University of Texas, \\ Austin, TX 78712, USA
}

\begin{abstract}
We consider the rational linear relations between real numbers whose squared trigonometric functions have rational values, angles we call "geodetic." We construct a convenient basis for the vector space over $\mathbb{Q}$ generated by these angles. Geodetic angles and rational linear combinations of geodetic angles appear naturally in Euclidean geometry; for illustration we apply our results to equidecomposability of polyhedra.
\end{abstract}

\section{Introduction}

Many well-known geometric objects involve angles that are irrational when measured in degrees or are irrational multiples of $\pi$ in radian measure. For instance, we might mention the dihedral angle $\alpha\left(\approx 70^{\circ} 31^{\prime} 44^{\prime \prime}\right)$ of the regular tetrahedron, whose supplement $\left(\approx 109^{\circ} 28^{\prime} 16^{\prime \prime}\right)$ is known to chemists as the carbon valence bond angle. A goodly number of these angles have the property that their six trigonometric functions have rational squares. For instance,

$\sin ^{2} \alpha=\frac{8}{9}, \quad \cos ^{2} \alpha=\frac{1}{9}, \quad \tan ^{2} \alpha=8, \quad \cot ^{2} \alpha=\frac{1}{8}, \quad \sec ^{2} \alpha=9, \quad \csc ^{2} \alpha=\frac{9}{8}$,

and, for the dihedral angle $\beta\left(\approx 144^{\circ} 44^{\prime} 8^{\prime \prime}\right)$ of the cuboctahedron,

$\sin ^{2} \beta=\frac{2}{3}, \quad \cos ^{2} \beta=\frac{1}{3}, \quad \tan ^{2} \beta=2, \quad \cot ^{2} \beta=\frac{1}{2}, \quad \sec ^{2} \beta=3, \quad \csc ^{2} \beta=\frac{3}{2}$.

\footnotetext{
* The first author's research was supported in part by NSF Grant No. DMS-9701444. The research of the second author was supported in part by NSF Grant No. DMS-9531584 and Texas ARP Grant 003658-152. The third author's research was supported in part by NSF Grant No. DMS-9626698 and Texas ARP Grant 003658-152.
} 
There are many additive relations between angles of this kind; for instance, $\alpha$ and $\beta$ satisfy $\alpha+2 \beta=2 \pi$. In this paper we essentially classify all such additive relations.

To be precise, we say that $\theta$ is a "pure geodetic angle" if any one (and therefore each) of its six squared trigonometric functions is rational (or infinite), and use the term "mixed geodetic angle" to mean a linear combination of pure geodetic angles with rational coefficients. The mixed geodetic angles form a vector space over the rationals and we shall find an explicit basis for this space. Finding a basis is tantamount to classifying all rational linear relations among mixed geodetic angles. By clearing denominators, rational linear relations among mixed geodetic angles are easily converted to additive relations among pure geodetic angles.

Another aim of this paper is to introduce an elegant notation for these angles which we hope will find general acceptance. Namely, if $0 \leq r \leq 1$ is rational we define

$$
\angle r=\sin ^{-1} \sqrt{r} \text {. }
$$

(We feel free to write these angles in either degrees or radians.) The well-known particular cases are:

$$
\angle 0=0^{\circ}=0, \quad \angle \frac{1}{4}=30^{\circ}=\frac{\pi}{6}, \quad \angle \frac{1}{2}=45^{\circ}=\frac{\pi}{4}, \quad \angle \frac{3}{4}=60^{\circ}=\frac{\pi}{3}, \quad \angle 1=90^{\circ}=\frac{\pi}{2} .
$$

We extend this notation for all integers $n$, by writing

$$
\angle n=90 n^{\circ}=\frac{n \pi}{2}, \quad \angle(n+r)=\angle n+\angle r .
$$

Our basis contains certain angles $\langle p\rangle_{d}$ for prime $p$ and square-free positive $d$. If $p>2$ or if $p=2$ and $d \equiv 7(\bmod 8)$, then $\langle p\rangle_{d}$ is defined just when $-d$ is congruent to a square modulo $p$ and is found as follows. Express $4 p^{s}$ as $a^{2}+d b^{2}$ for the smallest possible positive $s$. Then

$$
\langle p\rangle_{d}=\frac{1}{s} \angle \frac{d b^{2}}{4 p^{s}}=\frac{1}{s} \sin ^{-1} \sqrt{\frac{d b^{2}}{4 p^{s}}}=\frac{1}{s} \cos ^{-1} \sqrt{\frac{a^{2}}{4 p^{s}}}=\frac{1}{s} \tan ^{-1} \sqrt{\frac{d b^{2}}{a^{2}}} .
$$

The expression is unique except when $d=1$ or 3 , when we make it so by demanding that $b$ be even (if $d=3$ ) or divisible by four (if $d=1$ ). (Some exercise in the notation is provided in Tables 1 and 2, which show the first few elements in the basis.) Our main result is then

Theorem 1. Every pure geodetic angle is uniquely expressible as a rational multiple of $\pi$ plus an integral linear combination of the angles $\langle p\rangle_{d}$. So the angles $\langle p\rangle_{d}$, supplemented by $\pi$ (or $\angle 1$ or $1^{\circ}$ ), form a basis for the space of mixed geodetic angles.

It is easy to find the representation of any pure geodetic angle $\theta$ in terms of the basis.

Theorem 2. If $\tan \theta=(b / a) \sqrt{d}$ for integers $a, b, d$, with square-free positive $d$ and with relatively prime $a$ and $b$, and if the prime factorization of $a^{2}+d b^{2}$ is $p_{1} p_{2} \cdots p_{n}$ (including multiplicity), then we have

$$
\theta=t \pi \pm\left\langle p_{1}\right\rangle_{d} \pm\left\langle p_{2}\right\rangle_{d} \pm \cdots \pm\left\langle p_{n}\right\rangle_{d}
$$

for some rational $t$. 
Table 1. Basis elements $\langle p\rangle_{d}$ for some $p$ and $d$.\# indicates that $(p)$ is prime in $\mathcal{O}_{d}$, while * indicates that $(p)$ ramifies.

\begin{tabular}{|c|c|c|c|c|c|c|c|c|c|c|c|c|c|c|c|}
\hline$d \backslash p$ & 2 & 3 & 5 & 7 & 11 & 13 & 17 & 19 & 23 & 29 & 31 & 37 & 41 & 43 & 47 \\
\hline 1 & $*$ & $\#$ & $\angle \frac{4}{5}$ & $\#$ & $\#$ & $\angle L_{13}^{4}$ & \begin{tabular}{|l|}
16 \\
17
\end{tabular} & $\#$ & $\#$ & $\angle \angle_{39}^{4}$ & $\#$ & $L_{37}^{36}$ & $L_{41}^{16}$ & $\#$ & \# \\
\hline 2 & * & $\angle \frac{2}{3}$ & $\#$ & $\#$ & $\angle \frac{2}{11}$ & $\#$ & $\angle \frac{8}{17}$ & $\angle \frac{18}{1.1}$ & $\#$ & $\#$ & $\#$ & $\#$ & $\angle \frac{32}{41}$ & $\angle \frac{18}{43}$ & \# \\
\hline 3 & H & * & H & $4 \frac{3}{7}$ & H & $\angle \frac{12}{13}$ & $\#$ & $\angle \frac{3}{19}$ & $\#$ & $\#$ & $\angle \frac{21}{31}$ & $\angle \frac{12}{37}$ & $\#$ & $\angle \frac{27}{43}$ & $\#$ \\
\hline 5 & $*$ & $\frac{1}{2}<\frac{5}{9}$ & $*$ & $\frac{1}{2} \angle \frac{45}{49}$ & $\#$ & $\#$ & $\#$ & $\#$ & $\frac{1}{2}<\frac{45}{529}$ & $\angle \frac{20}{29}$ & $\#$ & $\#$ & $\angle \frac{5}{41}$ & $\frac{1}{2}<\frac{445}{1840}$ & $\frac{1}{2}<\frac{2205}{2209}$ \\
\hline 6 & $*$ & $*$ & $\frac{1}{2}<\frac{24}{25}$ & $\angle \frac{9}{7}$ & $\frac{1}{2}<\frac{96}{121}$ & \# & $\#$ & $\#$ & $\#$ & $\frac{1}{2} / \frac{216}{841}$ & $/ \frac{6}{31}$ & $\#$ & $\#$ & $\#$ & $\#$ \\
\hline 7 & $\angle \frac{7}{8}$ & $\#$ & $\#$ & $*$ & $\angle \frac{7}{11}$ & $\#$ & $\#$ & $\#$ & $\angle \frac{7}{23}$ & $\angle \frac{28}{29}$ & $\#$ & $\angle \frac{28}{37}$ & $\#$ & $\angle \frac{7}{43}$ & $\#$ \\
\hline 10 & * & $\#$ & $*$ & $\frac{1}{2}<\frac{40}{49}$ & $\angle \frac{10}{17}$ & $\frac{1}{2}<\frac{1600}{1694}$ & $\#$ & $\angle \frac{10}{19}$ & $\frac{1}{2}<\frac{360}{52.11}$ & \# & $\#$ & $\frac{1}{2}<\frac{640}{1369}$ & $\angle \frac{40}{41}$ & $\#$ & $\frac{1}{2}<\frac{360}{2200}$ \\
\hline 11 & $\#$ & $\angle \frac{11}{12}$ & $\angle \frac{11}{20}$ & $\#$ & $*$ & $\#$ & $\#$ & $\#$ & $\angle \frac{11}{912}$ & $\#$ & $\angle \frac{9 ! 9}{124}$ & $\angle \frac{99}{148}$ & $\#$ & $\#$ & $4 \frac{11}{47}$ \\
\hline 13 & * & $\#$ & $\#$ & $\frac{1}{2}<\frac{13}{19}$ & $\frac{1}{2}<\frac{117}{121}$ & * & $\angle_{17}^{13}$ & $\frac{1}{2}<\frac{325}{301}$ & $\#$ & $\angle_{20}^{13}$ & ${ }_{2}^{1} \angle_{961}^{637}$ & $\#$ & $\#$ & $\#$ & $\begin{array}{l}1 \\
2\end{array}$ \\
\hline 14 & $*$ & $\frac{1}{4}<\frac{56}{81}$ & $\frac{1}{4}<\frac{501}{62 z}$ & $*$ & $\#$ & $\frac{1}{4}<\frac{4536}{284 t 61}$ & $\#$ & $\frac{1}{4}<\frac{21696}{13131321}$ & $\angle \frac{14}{23}$ & $\#$ & $\#$ & $\#$ & $\#$ & $\#$ & $\#$ \\
\hline 15 & $\frac{1}{2}<\frac{15}{16}$ & $*$ & $*$ & $H$ & \# & \# & $\frac{1}{2}<\frac{240}{289}$ & $\angle \frac{15}{19}$ & $\frac{1}{2}<\frac{240}{529}$ & $\#$ & $\angle \frac{16}{31}$ & $\#$ & $\#$ & $\#$ & $\frac{1}{2}<\frac{2100}{22019}$ \\
\hline 17 & $*$ & $\frac{1}{4}<\frac{17}{81}$ & $\#$ & $\frac{1}{4}<\frac{1377}{2401}$ & $\frac{1}{4}<\frac{3825}{14641}$ & $\frac{1}{2}<\frac{153}{109}$ & $*$ & $\#$ & $\frac{1}{4}<\frac{1874255}{279841}$ & $\#$ & $\frac{1}{4}<\frac{971137}{9232521}$ & $\#$ & $\#$ & $\#$ & $\#$ \\
\hline 19 & $\#$ & $\#$ & $1 \frac{19}{20}$ & $/ \frac{19}{28}$ & $\angle \frac{19}{44}$ & $\#$ & $\angle \angle \frac{19}{68}$ & * & $\angle \frac{19}{23}$ & $\#$ & $\#$ & $\#$ & $\#$ & $\angle \frac{171}{172}$ & $<\frac{19}{188}$ \\
\hline 21 & $*$ & * & $\frac{1}{2}<\frac{21}{25}$ & * & $\frac{1}{2}<\frac{21}{121}$ & $\#$ & $\frac{1}{2}<\frac{189}{289}$ & $\frac{1}{2}<\frac{336}{361}$ & \begin{tabular}{|l} 
\\
$\frac{1}{2}<\frac{525}{5225}$ \\
\end{tabular} & $\#$ & $\frac{1}{2}<\frac{336}{961}$ & $\angle \frac{21}{37}$ & $\frac{1}{2}<\frac{525}{1681}$ & \# & $\#$ \\
\hline 22 & $*$ & $\#$ & $\#$ & $\#$ & $*$ & $\frac{1}{2}<\frac{98}{109}$ & $\#$ & $\frac{1}{2}<\frac{352}{361}$ & $\quad \angle \frac{22}{23}$ & \begin{tabular}{|l|l|}
$\frac{1}{2}<\frac{702}{841}$ \\
\end{tabular} & $\angle \frac{22}{31}$ & $\#$ & \# & $\frac{1}{2}<\frac{14089}{1849}$ & $\angle \frac{22}{47}$ \\
\hline 23 & $\left|\frac{1}{3}<\frac{22}{32}\right|$ & $\frac{1}{3}<\frac{23}{27}$ & $\#$ & $\#$ & $\#$ & $\frac{8}{3} \angle \frac{818}{2197}$ & $\#$ & $\#$ & $*$ & $\frac{1}{3}<\frac{45 \times 18}{24389}$ & $\frac{1}{3}<\frac{2117}{29791}$ & $\#$ & $\frac{1}{3}<\frac{2.3557}{68921}$ & 吾 $\quad \#$ & $\frac{1}{3}<\frac{101947}{103823}$ \\
\hline
\end{tabular}

(We note that the denominator of $t$ will be a divisor of the class number of $\mathbb{Q}(\sqrt{-d})$.)

For example, for $\tan \theta=\frac{5}{4} \sqrt{3}$ we find $a^{2}+d b^{2}=16+75=91=7 \cdot 13$ and indeed $\theta=n \pi-\langle 7\rangle_{3}-\langle 13\rangle_{3}$.

Our results have an outstanding application. In 1900 Dehn [Deh] solved Hilbert's third problem [Sah] by giving a necessary condition for the mutual equidecomposability of polyhedra in terms of their dihedral angles, from which it follows easily that there are tetrahedra of equal volume which are not equidecomposable. In 1965 Sydler proved [Syd] that Dehn's criterion is also sufficient. For polyhedra with geodetic dihedral angles our Theorem 1 makes the Dehn-Sydler criterion effective. At the end of this paper we apply our theory to the non-snub Archimedean polyhedra (whose dihedral angles are all geodetic).

\section{Angles with Polyquadratic Tangents and the Splitting Theorem}

The addition formula for tangents enables us to show that the tangent of any sum of pure geodetic angles is a "polyquadratic number," that is a number of the form $\sqrt{a}+\sqrt{b}+$ $\sqrt{c}+\cdots$, with $a, b, c, \ldots$ rational. For instance, if $\tan \alpha=\sqrt{2} / 2$ and $\tan \beta=\sqrt{3} / 3$, then

$$
\begin{aligned}
& \tan (\alpha+\beta)=\frac{\sqrt{2} / 2+\sqrt{3} / 3}{1-\sqrt{2} \sqrt{3} / 6}=\frac{4}{5} \sqrt{2}+\frac{3}{5} \sqrt{3} \\
& \tan (\alpha-\beta)=\frac{4}{5} \sqrt{2}-\frac{3}{5} \sqrt{3} .
\end{aligned}
$$


Table 2. The values of some basis elements $\langle p\rangle_{d}$.

$\langle 5\rangle_{1}-\angle \frac{4}{5} \approx 63^{\circ} 26^{\prime} 6^{\prime \prime}$

$\langle 13\rangle_{1}=\angle \frac{4}{13} \approx 33^{\circ} 41^{\prime} 24^{\prime \prime}$

$\langle 17\rangle_{1}=\angle \frac{16}{17} \approx 75^{\circ} 57^{\prime} 50^{\prime \prime}$

$\langle 29\rangle_{1}=\angle \frac{4}{29} \approx 21^{\circ} 48^{\prime} 5^{\prime \prime}$

$\langle 37\rangle_{1}=\angle \frac{36}{37} \approx 80^{\circ} 32^{\prime} 16^{\prime \prime}$

$\langle 41\rangle_{1}=/ \frac{10}{41} \approx 38^{\circ} 39^{\prime} 35^{\prime \prime}$

$\langle 3\rangle_{2}=\angle \frac{2}{3} \approx 54^{\circ} 44^{\prime} 8^{\prime \prime}$

$\langle 11\rangle_{2}=\angle \frac{2}{11} \approx 25^{\circ} 14^{\prime \prime} 22^{\prime \prime}$

$\langle 17\rangle_{2}=L_{17}^{8} \approx 43^{\circ} 18^{\prime} 50^{\prime \prime}$

$\langle 19\rangle_{2}=\angle \frac{18}{19} \approx 76^{\circ} 44^{\prime} 14^{\prime \prime}$

$\langle 11\rangle_{2}=\angle \frac{32}{41} \approx 62^{\circ} 3^{\prime} 12^{\prime \prime}$

$\langle 43\rangle_{2}-\angle \frac{18}{43} \approx 40^{\circ} 18^{\prime} 55^{\prime \prime}$

$\langle 7\rangle_{3}=\angle \frac{3}{7} \approx 40^{\circ} 53^{\prime} 36^{\prime \prime}$

$\langle 13\rangle_{3}=\angle \frac{12}{13} \approx 73^{\circ} 53^{\prime} 52^{\prime \prime}$

$\langle 19\rangle_{3}=\angle \frac{3}{19} \approx 23^{\circ} 24^{\prime} 48^{\prime \prime}$

$\langle 31\rangle_{3}=\angle \frac{27}{31} \approx 68^{\circ} 56^{\prime} 54^{\prime \prime}$

$\langle 37\rangle_{3}=\angle \frac{12}{37} \approx 34^{\circ} 42^{\prime} 54^{\prime \prime}$

$\langle 43\rangle_{3}=\angle \frac{27}{43} \approx 52^{\cup} 24^{\prime} 39^{\prime \prime}$

$\langle 3\rangle_{5}=\frac{1}{2} \angle \frac{5}{9} \approx 24^{\circ} 5^{\prime} 41^{\prime \prime}$

$\langle 7\rangle_{5}=\frac{1}{2} \angle \frac{45}{49} \approx 36^{\circ} 11^{\prime} 57^{\prime \prime}$

$\langle 23\rangle_{5}-\frac{1}{2} \angle \frac{45}{529} \approx 8^{\circ} 28^{\prime} 43^{\prime \prime}$

$\langle 29\rangle_{5}=\angle \frac{20}{29} \approx 56^{\circ} 8^{\prime} 44^{\prime \prime}$

$\langle 41\rangle_{5}=\angle \frac{5}{41} \approx 20^{\circ} 26^{\prime} 22^{\prime \prime}$

$\langle 43\rangle_{5}=\frac{1}{2} \angle \frac{405}{1849} \approx 13^{\circ} 57^{\prime} 10^{\prime \prime}$

$\langle 47\rangle_{5}=\frac{1}{2} \angle \frac{2205}{2209} \approx 43^{\circ} 46^{\prime} 50^{\prime \prime}$

$\langle 5\rangle_{0}-\frac{1}{2} \angle \frac{24}{25} \approx 39^{\circ} 13^{\prime} 53^{\prime \prime}$

$\langle 7\rangle_{6}=\angle \frac{6}{7} \approx 67^{\circ} 47^{\prime} 32^{\prime \prime}$

$\langle 11\rangle_{6}=\frac{1}{2} \angle \frac{90}{121} \approx 31^{\circ} 28^{\prime} 56^{\prime \prime}$

$\langle 29\rangle_{6}=\frac{1}{2} \angle \frac{216}{841} \approx 15^{\circ} 13^{\prime} 31^{\prime \prime}$

$\langle 31\rangle_{6}=\angle \frac{6}{31} \approx 26^{\circ} 6^{\prime}\left(0^{\prime \prime}\right.$

$\langle 2\rangle_{7}-\angle \frac{7}{8} \approx 69^{\circ} 17^{\prime} 43^{\prime \prime}$

$\langle 11\rangle_{7}=\angle \frac{7}{11} \approx 52^{\circ} 54^{\prime} 48^{\prime \prime}$

$\langle 23\rangle_{7}=\angle \frac{7}{23} \approx 33^{\circ} 28^{\prime} 56^{\prime \prime}$

$\langle 29\rangle_{7}=\angle \frac{28}{29} \approx 79^{\circ} 17^{\prime} 54^{\prime \prime}$

$\langle 37\rangle_{7}=/ \frac{28}{37} \approx 67^{\circ} 26^{\prime} 57^{\prime \prime}$

$\langle 43\rangle_{7}-\angle \frac{7}{43} \approx 23^{\circ} 47^{\prime} 44^{\prime \prime}$ $\langle 7\rangle_{10}=\frac{1}{2} \angle \frac{40}{10} \approx 32^{\circ} 18^{\prime} 42^{\prime \prime}$

$\langle 11\rangle_{10}-\angle \frac{10}{11} \approx 72^{\circ} 27^{\prime} 6^{\prime \prime}$

$\langle 13\rangle_{10}=\frac{1}{2} \angle \frac{160}{169} \approx 38^{\circ} 19^{\prime} 44^{\prime \prime}$

$\langle 19\rangle_{10}=\angle \frac{10}{10} \approx 46^{\circ} 30^{\prime} 31^{\prime \prime}$

$\langle 23\rangle_{10}=\frac{1}{2} \angle \frac{360}{529} \approx 27^{\circ} 47^{\prime} 29^{\prime \prime}$

$\langle 37\rangle_{10}=\frac{1}{2} / \frac{6440}{13(9)} \approx 21^{\circ} 34^{\prime} 6^{\prime \prime}$

$\left.\langle 41\rangle_{10}=\angle \frac{40}{41} \approx 81^{\circ}\right)^{\prime} 54^{\prime \prime}$

$\langle 47\rangle_{10}=\frac{1}{2} \angle \frac{360}{2200} \approx 11^{\circ} 54^{\prime} 17^{\prime \prime}$

$\langle 3\rangle_{11}=\angle_{12}^{11} \approx 73^{\circ} 13^{\prime} 17^{\prime \prime}$

$\langle 5\rangle_{11}=\angle \frac{11}{20} \approx 47^{\circ} 52^{\prime} 11^{\prime \prime}$

$\langle 23\rangle_{11}=\angle \frac{11}{02} \approx 20^{\circ} 13^{\prime} 16^{\prime \prime}$

$\langle 31\rangle_{11}=\angle \frac{99}{124} \approx 63^{\circ} 19^{\prime} 11^{\prime \prime}$

$\langle 37\rangle_{11}=\angle \frac{199}{148} \approx 54^{\circ} 52^{\prime} 21^{\prime \prime}$

$\langle 47\rangle_{11}=\angle \frac{11}{47} \approx 28^{\circ} 55^{\prime} 57^{\prime \prime}$

$\langle 7\rangle_{13}=\frac{1}{2} / \frac{13}{49} \approx 15^{\circ} 30^{\prime} 5^{\prime \prime}$

$\langle 11\rangle_{13}-\frac{1}{2}<\frac{117}{121} \approx 39^{\circ} 45^{\prime} 44^{\prime \prime}$

$\langle 17\rangle_{13}=\angle \frac{13}{17} \approx 60^{\circ} 58^{\prime} 58^{\prime \prime}$

$\langle 19\rangle_{13}=\frac{1}{2} \angle \frac{325}{361} \approx 35^{\circ} 47^{\prime} 45^{\prime \prime}$

$\langle 29\rangle_{13}=\angle \frac{13}{29} \approx 42^{\circ} 1^{\prime} 52^{\prime \prime}$

$\langle 31\rangle_{13}=\frac{1}{2} \angle \frac{637}{661} \approx 27^{\circ} 15^{\prime} 7^{\prime \prime}$

$\langle 17\rangle_{13}=\frac{1}{2} \angle \frac{1053}{2200} \approx 21^{\circ} 19^{\prime} 55^{\prime \prime}$

$\langle 3\rangle_{14}=\frac{1}{4} \angle \frac{56}{81} \approx 14^{\circ} 3^{\prime} 46^{\prime \prime}$

$\langle 5\rangle_{14}=\frac{1}{4} \angle \frac{504}{625} \approx 15^{\mathrm{n}} 58^{\prime} 27^{\prime \prime}$

$\langle 13\rangle_{14}=\frac{1}{4} \angle \frac{4536}{28561} \approx 5^{\circ} 52^{\prime} 17^{\prime \prime}$

$\langle 19\rangle_{14}=\frac{1}{4} / \frac{24690}{130321} \approx 6^{\circ} 27^{\prime} 5^{\prime \prime}$

$\langle 23\rangle_{11}-\angle \frac{14}{23} \approx 51^{\circ} 16^{\prime} 41^{\prime \prime}$

$\langle 2\rangle_{15}=\frac{1}{2} \angle \frac{15}{16} \approx 37^{\circ} 45^{\prime} 40^{\prime \prime}$

$\langle 17\rangle_{15}=\frac{1}{2} \angle \frac{240}{280} \approx 32^{\circ} 50^{\prime} 32^{\prime \prime}$

$\langle 19\rangle_{15}=\angle{ }_{19}^{15} \approx 62^{\circ} 41^{\prime} 18^{\prime \prime}$

$\langle 23\rangle_{15}=\frac{1}{2} \angle \frac{240}{520} \approx 21^{\circ} 10^{\prime} 17^{\prime \prime}$

$\langle 31\rangle_{15}=\angle \frac{15}{31} \approx 44^{\circ} 4^{\prime} 33^{\prime \prime}$

$\langle 47\rangle_{15}=\frac{1}{2}<\frac{2160}{2209} \approx 40^{\circ} 43^{\prime} 2^{\prime \prime}$

$\langle 3\rangle_{17}-\frac{1}{1} \angle \frac{17}{81} \approx 6^{\circ} 48^{\prime} 59^{\prime \prime}$

$\langle 7\rangle_{17}-\frac{1}{4} \angle \frac{1377}{2401} \approx 12^{\circ} 18^{\prime} 24^{\prime \prime}$

$\langle 11\rangle_{17}=\frac{1}{4} \angle \frac{3825}{14641} \approx 7^{\circ} 41^{\prime} 5^{\prime \prime}$

$\langle 13\rangle_{17}=\frac{1}{2} \angle \frac{153}{169} \approx 36^{\circ} 2^{\prime} 24^{\prime \prime}$

$\langle 23\rangle_{17}=\frac{1}{4} \angle \frac{187455}{279841} \approx 13^{\circ} 43^{\prime} 51^{\prime \prime}$

$\langle 31\rangle_{17}=\frac{1}{4} / \frac{907137}{923521} \approx 20^{\circ} 35^{\prime} 11^{\prime \prime}$

$\langle 5\rangle_{19}=\angle \frac{19}{20} \approx 77^{\circ} 4^{\prime} 45^{\prime \prime}$

$\langle 7\rangle_{19}=\angle \frac{19}{28} \approx 55^{\circ} 27^{\prime} 45^{\prime \prime}$

$\langle 11\rangle_{19}=\angle_{4}^{19} \approx 41^{\circ} 4^{\prime} 53^{\prime \prime}$

$\langle 17\rangle_{19}=\angle \frac{19}{68} \approx 31^{\circ} 54^{\prime} 38^{\prime \prime}$

$\langle 23\rangle_{19}=\angle \frac{19}{23} \approx 65^{\circ} 21^{\prime} 10^{\prime \prime}$

$\langle 43\rangle_{19}=\angle \frac{171}{172} \approx 85^{\circ} 37^{\prime} 37^{\prime \prime}$

$\langle 47\rangle_{19}=\angle \frac{19}{188} \approx 18^{\circ} 32^{\prime} 11^{\prime \prime}$

$\langle 5\rangle_{21}=\frac{1}{2} \angle \frac{21}{25} \approx 33^{\circ} 12^{\prime} 39^{\prime \prime}$

$\langle 11\rangle_{21}=\frac{1}{2}<\frac{21}{121} \approx 12^{\circ} 18^{\prime} 36^{\prime \prime}$

$\langle 17\rangle_{21}=\frac{1}{2} \angle \frac{189}{289} \approx 26^{\circ} 59^{\prime} 3^{\prime \prime}$

$\langle 19\rangle_{21}=\frac{1}{2}<\frac{336}{301} \approx 37^{\circ} 22^{\prime} 16^{\prime \prime}$

$\langle 23\rangle_{21}=\frac{1}{2} \angle \frac{25}{529} \approx 42^{\circ} 30^{\prime \prime} 21^{\prime \prime}$

$\langle 31\rangle_{21}=\frac{1}{2} \angle \frac{336}{961} \approx 18^{\circ} 7^{\prime} 29^{\prime \prime}$

$\langle 37\rangle_{21}=\angle \frac{21}{37} \approx 48^{\circ} 53^{\prime} 0^{\prime \prime}$

$\langle 11\rangle_{21}=\frac{1}{2} \angle \frac{525}{1681} \approx 16^{\circ} 59^{\prime} 17^{\prime \prime}$

$\langle 13\rangle_{22}=\frac{1}{2} \angle \frac{88}{169} \approx 23^{\circ} 5^{\prime} 36^{\prime \prime}$

$\langle 19\rangle_{22}=\frac{1}{2} \angle \frac{3.52}{361} \approx 40^{n} 27^{\prime} 28^{\prime \prime}$

$\langle 23\rangle_{22}=\angle \frac{22}{2.3} \approx 77^{\circ} 57^{\prime} 5.53^{\prime \prime}$

$\langle 29\rangle_{22}=\frac{1}{2} \angle \frac{792}{841} \approx 38^{\circ} 0^{\prime} 58^{\prime \prime}$

$\langle 31\rangle_{22}=\angle \frac{22}{31} \approx 57^{\circ} 23^{\prime} 49^{\prime \prime}$

$\langle 43\rangle_{22}=\frac{1}{2} \angle \frac{1408}{1849} \approx 30^{\circ} 22^{\prime} 59^{\prime \prime}$

$\langle 47\rangle_{22}=\angle \frac{22}{47} \approx 43^{\circ} 10^{\prime} 11^{\prime \prime}$

$\langle 2\rangle_{2.3}=\frac{1}{3} \angle \frac{23}{32} \approx 19^{\circ} 19^{\prime} 27^{\prime \prime}$

$\langle 3\rangle_{23}=\frac{1}{3} \angle \frac{23}{27} \approx 22^{\circ} 27^{\prime} 15^{\prime \prime}$

$\langle 13)_{23}-\frac{1}{3} \angle \frac{828}{2107} \approx 12^{\circ} 37^{\prime} 28^{\prime \prime}$

$\langle 29\rangle_{23}=\frac{1}{3} \angle \frac{4508}{24389} \approx 8^{\circ} 29^{\prime} 16^{\prime \prime}$

$\langle 31\rangle_{23}=\frac{1}{3} \angle \frac{207}{20791} \approx 1^{\mathrm{n}} 35^{\prime} 38^{\prime \prime}$

$\langle 41\rangle_{23}=\frac{1}{3} \angle \frac{23552}{6.3921} \approx 11^{\circ} 55^{\prime} 27^{\prime \prime}$

$\langle 47\rangle_{23}=\frac{1}{3} \angle \frac{103247}{103823} \approx 28^{\circ}: 34^{\prime} 34^{\prime \prime}$ 
We now suppose the sum of a number of pure geodetic angles is an integral multiple of $\pi$; let us say $\alpha_{1}+\alpha_{2}+\cdots+\beta_{1}+\beta_{2}+\cdots=m \pi$, where we have chosen the notation so that the tangents of $\alpha_{1}, \alpha_{2}, \ldots$ are in $\mathbb{Q}\left(\sqrt{d_{1}}, \ldots, \sqrt{d_{n}}\right)$ and those of $\beta_{1}, \beta_{2}, \ldots$ are in $\sqrt{d} \mathbb{Q}\left(\sqrt{d_{1}}, \ldots, \sqrt{d_{n}}\right)$, where $\sqrt{d} \notin \mathbb{Q}\left(\sqrt{d_{1}}, \ldots, \sqrt{d_{n}}\right)$. Then, by the addition and substraction formulas for tangents, $\tan \left(\alpha_{1}+\alpha_{2}+\cdots+\beta_{1}+\beta_{2}+\cdots\right)$ and $\tan \left(\alpha_{1}+\alpha_{2}+\cdots-\right.$ $\left.\beta_{1}-\beta_{2}-\cdots\right)$ will be of the form $a+b \sqrt{d}$ and $a-b \sqrt{d}$ where $a, b \in \mathbb{Q}\left(\sqrt{d_{1}}, \ldots, \sqrt{d_{n}}\right)$. However, by assumption $a+b \sqrt{d}=0$, so $a=b=0$, from which it follows that $\alpha_{1}+\alpha_{2}+\cdots-\beta_{1}-\beta_{2}-\cdots$ is also an integral multiple of $\pi$. Adding and subtracting we deduce that the two subsums $\alpha_{1}+\alpha_{2}+\cdots$ and $\beta_{1}+\beta_{2}+\cdots$ are integral multiples of $\pi / 2$. Combining this argument with induction on $n$ we obtain

Theorem 3 (The Splitting Theorem). If the value of a rational linear combination of pure geodetic angles is a rational multiple of $\pi$, then so is the value of its restriction to those angles whose tangents are rational multiples of any given square root.

We remark that the same method can be used to show that any angle whose tangent is polyquadratic is a mixed geodetic angle. For suppose $\alpha$ is an angle whose tangent is polyquadratic, with $\tan \alpha \in \sqrt{d_{0}} \mathbb{Q}\left(\sqrt{d_{1}}, \ldots, \sqrt{d_{n}}\right)$. So $\tan \alpha=z_{1}+z_{2} \sqrt{d_{n}}$, where $z_{j} \in \sqrt{d_{0}} \mathbb{Q}\left(\sqrt{d_{1}}, \ldots, \sqrt{d_{n-1}}\right)$. Choose $\alpha^{\prime}$ such that $\tan \alpha^{\prime}=z_{1}-z_{2} \sqrt{d_{n}}$, and define $\gamma=\alpha+\alpha^{\prime}$ and $\delta=\alpha-\alpha^{\prime}$. It follows that

$$
\begin{aligned}
& \tan \gamma=\frac{\tan \alpha+\tan \alpha^{\prime}}{1-\tan \alpha \tan \alpha^{\prime}}=\frac{2 z_{1}}{1-z_{1}^{2}+d_{n} z_{2}^{2}} \in \sqrt{d_{0}} \mathbb{Q}\left(\sqrt{d_{1}}, \ldots, \sqrt{d_{n-1}}\right) \\
& \tan \delta=\frac{\tan \alpha-\tan \alpha^{\prime}}{1+\tan \alpha \tan \alpha^{\prime}}=\frac{2 z_{2} \sqrt{d_{n}}}{1+z_{1}^{2}-d_{n} z_{2}^{2}} \in \sqrt{d_{0} d_{n}} \mathbb{Q}\left(\sqrt{d_{1}}, \ldots, \sqrt{d_{n-1}}\right)
\end{aligned}
$$

Repetition of this technique justifies our claim. We leave to the reader the exercise of applying this technique to the case of $\tan \alpha=\sqrt{6}+\sqrt{3}+\sqrt{2}+1$, obtaining $4 \alpha=$ $\angle\left(1+\frac{441}{457}\right)+\angle \frac{432}{457}+\angle \frac{96}{457}+\angle \frac{2592}{4113}$.

\section{Størmer Theory and Its Generalization}

The Splitting Theorem reduces the study of the rational linear relationships between angles of the form $\tan ^{-1}((b / a) \sqrt{d})$ to those with a fixed $d$. These angles are the arguments of algebraic integers $a+b \sqrt{-d}$, and their theory is essentially the factorization theory of numbers in $\mathcal{O}_{d}$, the ring of algebraic integers of $\mathbb{Q}(\sqrt{-d})$ [Pol]. The method was first used by Størmer [Stø] (in the case $d=1$ ) who classified the additive relations between the arctangents of rational numbers using the unique factorization of Gaussian integers. (See also [Con].) We recall Størmer's analysis of the case $d=1$ and then generalize it to arbitrary $d$, which will prove Theorems 1 and 2 .

It is known that the Gaussian integers have unique factorization up to multiplication by the four units: $1,-1, i,-i$. It is also known how each rational prime $p$ factorizes in the Gaussian integers. Namely: (1) if $p \equiv-1(\bmod 4)$, then $p$ remains prime; (2) if $p \equiv+1(\bmod 4)$, then $p=a^{2}+b^{2}$ is the product of the distinct Gaussian primes $a+i b$ 
and $a-i b$ (for uniqueness we choose $a$ odd, $b$ even, both positive); and (3) $2=-i(1+i)^{2}$ "ramifies," that is to say it is (a unit times) the square of a Gaussian prime.

Now let $\kappa=\pi_{1} \pi_{2} \pi_{3} \cdots$ be the prime factorization of a Gaussian integer $\kappa$. Then plainly $\arg (\kappa) \equiv \arg \left(\pi_{1}\right)+\arg \left(\pi_{2}\right)+\arg \left(\pi_{3}\right)+\cdots(\bmod 2 \pi)$. So the arguments of Gaussian primes (together with $\pi$ ) span the subspace of mixed geodetic angles generated by the pure geodetic angles with rational tangent. However,

(1) If $p=4 k-1$, then $\arg (p)=0$ and can be ignored.

(2) If $p=4 k+1=a^{2}+b^{2}$, then $\arg (a+b i)+\arg (a-b i)=0$. We define $\langle p\rangle_{1}=\arg (a+b i)=-\arg (a-b i)$.

(3) $\arg (1+i)=\pi / 4$.

(4) The arguments of the units are multiples of $\pi / 2$, and so are multiples of $\arg (1+i)$.

Thus the argument of any Gaussian integer is an integral linear combination of $\pi / 4$ and the angles $\langle p\rangle_{1}$, with $p \equiv 1(\bmod 4)$.

It is also easy to see that $\pi$ and the $\langle p\rangle_{1}$ 's are rationally independent. Otherwise some integral linear combination of the $\langle p\rangle_{1}$ 's would be an integral multiple of $\pi$. However, suppose for instance that $2\left\langle p_{1}\right\rangle_{1}-3\left\langle p_{2}\right\rangle_{1}+5\left\langle p_{3}\right\rangle_{1}=0$. The left-hand side is the argument of $\pi_{1}^{2} \bar{\pi}_{2}^{3} \pi_{3}^{5}$ which must therefore be a real number and hence should be equal to its conjugate $\bar{\pi}_{1}^{2} \pi_{2}^{3} \bar{\pi}_{3}^{5}$; but this contradicts the unique factorization of Gaussian integers.

The analogue of the Størmer theory for the general case is complicated by the fact that some elements of $\mathcal{O}_{d}$ may not have unique factorization. However, the ideals do. Instead of assigning arguments to numbers, we simply assign an angle to each ideal II by the rule

$$
\arg (\mathbb{I})= \begin{cases}\arg (\kappa) & \text { if } \mathbb{I}=(\kappa) \text { is principal; } \\ (1 / s) \arg \left(\mathbb{I}^{s}\right) & \text { if } \mathbb{I} \text { is not principal, }\end{cases}
$$

where $s$ is the smallest exponent for which $\mathbb{I}^{s}$ is a principal ideal, and $(\kappa)$ denotes the principal ideal generated by $\kappa$. Recall that for every $d$ the ideal class group is finite, so such an $s$ exists for every ideal, and $s$ divides the class number of $\mathcal{O}_{d}$. Since the generator of a principal ideal is unique up to multiplication by a unit, we take the argument of an ideal to be defined only modulo the argument of a unit divided by the class number of $\mathcal{O}_{d}$. This ambiguity is always a rational multiple of $\pi$.

Let $c_{d}$ be the class number of $\mathcal{O}_{d}$. For every ideal $\mathbb{I}$, principal or not, we have that the ideal argument $\arg (\mathbb{I})$ is equal to $1 / c_{d}$ times the (ordinary) argument of the generator of the principal ideal $\mathbb{I}^{c_{d}}$, up to the ambiguity in the definition of ideal arguments. It follows that, for general ideals $\mathbb{I}$ and $\mathbb{J}$,

$$
\arg (\mathbb{I} \mathbb{J})=\arg (\mathbb{I})+\arg (\mathbb{J})
$$

(modulo the ambiguity), since the (ordinary) argument of the generator of $(\mathbb{I} \mathbb{J})^{c_{d}}$ is the argument of the generator of $\mathbb{I}^{c_{d}}$ plus the argument of the generator of $\mathbb{J}^{c_{d}}(\bmod \pi)$. Thus the argument of any ideal (and in particular the principal ideal generated by any algebraic integer) is an integral linear combination of the arguments of the prime ideals (modulo the ambiguity). What remains is to determine the nontrivial arguments of prime ideals. As in the case $d=1$, there will be one such angle for each rational prime $p$ for which ( $p$ ) splits as the product of distinct ideals. 
We illustrate the procedure by working in $\mathcal{O}_{5}$, for which the ideal factorizations of the first few rational primes are:

$$
\begin{aligned}
& (2)=(2,1+\sqrt{-5})^{2} \\
& (3)=(3,1+\sqrt{-5})(3,1-\sqrt{-5}) \\
& (5)=(\sqrt{-5})^{2} \\
& (7)=(7,3+\sqrt{-5})(7,3-\sqrt{-5}) \\
& (11)=(11) \\
& (13)=(13) \\
& (17)=(17) \\
& (19)=(19) \\
& (23)=(23,22+3 \sqrt{-5})(23,22-3 \sqrt{-5}), \\
& (29)=(3+2 \sqrt{-5})(3-2 \sqrt{-5}) .
\end{aligned}
$$

(This list may be obtained using Theorems 5 and 6, below.) Here $(x, y)$ denotes the ideal generated by $x$ and $y$. The reader will see that (2) ramifies as the square of a nonprincipal ideal and (5) as the square of a principal ideal, (3), (7), and (23) split into products of nonprincipal ideals, (29) splits as the product of distinct principal ideals, while (11), (13), (17), and (19) remain prime. Notice that, as the example shows, every ideal of $\mathcal{O}_{d}$ can be generated by at most two numbers, and $(p)$ can be written as a product of at most two prime ideals, for any rational prime $p$.

As in the Størmer case the principal ideals generated by rational primes that remain prime have argument zero and can be ignored. We also ignore those that ramify, since their angles will be rational multiples of $\pi$. Otherwise we define $\langle p\rangle_{d}$ to be the argument of one of the two ideal factors of $(p)$, making it unique by requiring $0<\langle p\rangle_{d}<\pi / 2$ if the factors of $(p)$ are principal and $0<\langle p\rangle_{d}<\pi / 4$ if not.

To illustrate this we determine $\langle 3\rangle_{5}$. The ideal factors of (3) are nonprincipal so we square them:

$$
\begin{aligned}
(3,1+\sqrt{-5})^{2} & =\left(3^{2}, 3(1+\sqrt{-5}),(1+\sqrt{-5})^{2}\right) \\
& =(9,3+3 \sqrt{-5},-4+2 \sqrt{-5}),
\end{aligned}
$$

which reduces to $(2-\sqrt{-5})$. Similarly, $(3,1-\sqrt{-5})^{2}=(2+\sqrt{-5})$. So $\langle 3\rangle_{5}=$ $\frac{1}{2} \arg (2+\sqrt{-5})=\frac{1}{2} \tan ^{-1}\left(\frac{1}{2} \sqrt{5}\right)=\frac{1}{2} \angle \frac{5}{9}$.

In the general case ( $d$ an arbitrary square-free positive integer) the previously described procedure assigns an angle $\langle p\rangle_{d}$ to every rational prime $p$ for which $(p)$ splits as the product of two distinct prime ideals $\mathbb{I}$ and $\mathbb{J}$. Let $s$ be the smallest integer for which $\mathbb{I}^{s}$ (and therefore $\mathbb{J}^{s}$ ) is principal. Recall that the elements of $\mathcal{O}_{d}$ are of the form $a / 2+(b / 2) \sqrt{-d}$, where $a$ and $b$ are rational integers. If $d \not \equiv 3(\bmod 4)$, then $a$ and $b$ must be even; if $d \equiv 3(\bmod 4)$, then $a$ and $b$ are either both even or both odd. We can therefore write

$$
\mathbb{I}^{s}=\left(\frac{a}{2}+\frac{b}{2} \sqrt{-d}\right), \quad \mathbb{J}^{s}=\left(\frac{a}{2}-\frac{b}{2} \sqrt{-d}\right),
$$


where we can distinguish between $\mathbb{I}$ and $\mathbb{J}$ by supposing that $a$ and $b$ are positive. We take

$$
\langle p\rangle_{d}=\frac{1}{s} \tan ^{-1}\left(\frac{b}{a} \sqrt{d}\right)=\frac{1}{s} \angle \frac{b^{2} d}{a^{2}+b^{2} d} .
$$

The above defines the angles $\langle p\rangle_{d}$ uniquely for all $d$ other than 1 and 3 , because then the only units are \pm 1 , so that the only generators of $\mathbb{I}^{s}$ and $\mathbb{J}^{s}$ are the four numbers $\pm a / 2 \pm(b / 2) \sqrt{d}$. When $d=1$ we have the additional unit $i$ which effectively allows us to interchange $a$ and $b$ : we then achieve uniqueness by demanding that the generators of $\mathbb{I}$ be $a+b i$ with $a$ and $b$ positive integers with $b$ even. In the case $d=3$ the field has six units and the corresponding condition is that the generators of $\mathbb{I}$ should have the form $a+b \sqrt{-3}$ where $a$ and $b$ are positive integers.

Theorem 4. For fixed square-free positive d, consider the subspace of mixed geodetic angles generated by arctangents of rational multiples of $\sqrt{d}$. This subspace is spanned by $\pi$ and the nonzero angles of the form $\langle p\rangle_{d}$, where $p$ ranges over the rational primes for which ( $p$ ) splits as a product of distinct ideals in $\mathcal{O}_{d}$.

Proof. The proof is essentially that of the Størmer decomposition, only substituting the arguments of ideals for the arguments of algebraic integers.

Combining Theorem 4 with the Splitting Theorem, and by the rational independence of $\pi$ and the $\langle p\rangle_{d}$ 's for any fixed $d$ (the independence can be proved similarly as was shown in the case $d=1$ ), we obtain Theorem 1 .

Proof of Theorem 2. Recall that if $\mathbb{I}$ is any ideal (principal or not) in $\mathcal{O}_{d}$, then $\overline{I I}$ is a principal ideal with a positive integer generator that we call the norm of $\mathbb{I}$, and that norms are multiplicative [Pol]. From this it follows that the prime ideals are precisely the factors of $(p)$, with $p$ ranging over the rational primes, and that every prime ideal that is not generated by a rational prime has rational prime norm.

Now suppose that $\tan (\theta)=(b / a) \sqrt{d}$ with square-free positive $d$ and with relatively prime $a$ and $b$. Consider the factorization of the principal ideal $\mathbb{I}=(a+b \sqrt{-d})$. If $\mathbb{I}=\pi_{1} \pi_{2} \cdots \pi_{n}$, where each ideal $\pi_{i}$ is prime, then none of the $\pi_{i}$ 's are generated by rational primes, insofar as $a$ and $b$ are relatively prime. Thus each $\pi_{i}$ satisfies $\pi_{i} \bar{\pi}_{i}=\left(q_{i}\right)$ for some rational prime $q_{i}$. On the other hand, we have

$$
\left(p_{1}\right)\left(p_{2}\right) \cdots\left(p_{n}\right)=\left(a^{2}+b^{2} d\right)=\mathbb{I} \overline{\mathbb{I}}=\pi_{1} \bar{\pi}_{1} \cdots \pi_{n} \bar{\pi}_{n}
$$

So, after a suitable permutation of the indices $1, \ldots, n$ on the right side of (15) we have $\pi_{i} \bar{\pi}_{i}=\left(p_{i}\right)$, and so the argument of $\pi_{i}$ is $\pm\left\langle p_{i}\right\rangle_{d}$, for every $i$. However, $\theta=$ $\arg (a+b \sqrt{-d})=\arg (\mathbb{I})$ is the sum of the arguments of the $\pi_{i}$ 's, up to a rational multiple of $\pi$ that comes from the ambiguity in the definition of the argument of an ideal.

All that remains is to identify the pairs $(p, d)$ for which $\langle p\rangle_{d}$ is defined. The following theorems give the criteria. These criteria may be easily implemented, by hand for small $d$ 
and $p$, and by computer for larger values. The theorems themselves are standard results, and we leave the proofs to the reader.

Theorem 5. Let $p$ be an odd rational prime. The ideal $(p)$ of $\mathcal{O}_{d}$ splits as a product of distinct ideals if and only if we can write

$$
4 p^{s}=a^{2}+b^{2} d
$$

for integers $a$ and $b$ (neither a multiple of $p$ ) and for an exponent $s$ that divides the class number of $\mathcal{O}_{d}$. If $d \not \equiv 3(\bmod 4)$, or if $d=3$, then the factor of 4 is unnecessary, and the criterion for splitting reduces to

$$
p^{s}=a^{2}+b^{2} d
$$

for $a$ and $b$ nonzero $(\bmod p)$. The ideal $(p)$ is prime in $\mathcal{O}_{d}$ if and only if $-d$ is not equal to a square modulo $p$. If $(p)$ is not prime and does not split, then $(p)$ ramifies.

Theorem 5 gives criteria for all odd primes. The prime $p=2$ is somewhat different. Since both 0 and 1 are squares, every $-d$ is congruent to a square modulo 2 . However, there are values of $d$ for which (2) is prime.

Theorem 6. If $d \not \equiv 3(\bmod 4)$, then $(2)$ ramifies in $\mathcal{O}_{d}$. If $d \equiv 3(\bmod 8)$, then $(2)$ is prime. If $d \equiv 7(\bmod 8)$, then $(2)$ splits and we can write a power of 2 as $a^{2}+b d^{2}$.

\section{Applications to the Dehn-Sydler Criterion of Archimedean Polyhedra}

The Dehn invariant of a polyhedron whose $i$ th edge has length $\ell_{i}$ and dihedral angle $\theta_{i}$ is the formal expression $\sum_{i} \ell_{i} V\left[\theta_{i}\right]$ where the "vectors" $V\left[\theta_{i}\right]$ are subject to the relations

$$
V[r \theta+s \varphi]=r V[\theta]+s V[\varphi], \quad V[r \pi]=0,
$$

for all rational numbers $r$ and $s$. The $V[\theta]$ 's satisfy the same rational linear relations satisfied by the angles $\theta$ in the rational vector space they generate, together with the additional relation $V[\pi]=0$; however, we allow their coefficients to be arbitrary real numbers.

If every dihedral angle $\theta$ of a polyhedron is geodetic we can write

$$
\theta=r \pi+r_{1}\left\langle p_{1}\right\rangle_{d_{1}}+\cdots+r_{j}\left\langle p_{j}\right\rangle_{d_{j}}
$$

for rational numbers $r, r_{1}, \ldots, r_{j}$, so

$$
V[\theta]=r_{1} V\left[\left\langle p_{1}\right\rangle_{d_{1}}\right]+\cdots+r_{j} V\left[\left\langle p_{j}\right\rangle_{d_{j}}\right] .
$$

If the edge lengths of the polyhedron are rational its Dehn invariant will then be a rational linear combination of the $V\left[\langle p\rangle_{d}\right]$ 's.

It can be easily checked that each face of an Archimedean polyhedron (other than the snub cube and snub dodecahedron) is orthogonal to a rotation axis of one of the Platonic 


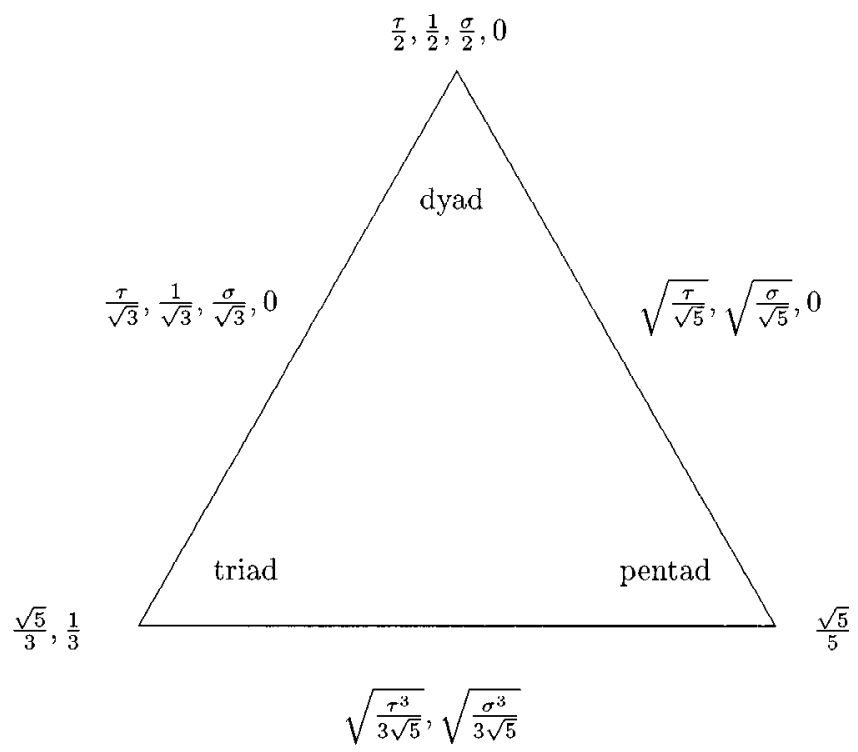

Fig. 1. Cosines of angles between axes of fixed rotational symmetry (shown at corners), and between axes of different rotational symmetry (shown at edges).

solids, and the rotation groups of all the Platonic solids are contained in the cube group $\mathcal{C}$ and icosahedral group $\mathcal{I}$. It follows that the dihedral angles of all these polyhedra are found among the supplements of the angles between the rotation axes of $\mathcal{C}$ and $\mathcal{I}$.

We now concentrate on $\mathcal{I}$. Let $\tau=(1+\sqrt{5}) / 2$ and $\sigma=\tau^{-1}=\tau-1$. The 12 vectors whose coordinates are cyclic permutations of $0, \pm 1, \pm \tau$ lie along the pentad axes. Similarly the 20 vectors obtained by cyclicly permutating $\pm 1, \pm 1, \pm 1$ and $0, \pm \tau, \pm \sigma$ lie along the triad axes, and the 30 cyclic permutations of $\pm 2,0,0$ and $\pm 1, \pm \sigma, \pm \tau$ lie along the dyad ones. The cosines of the angles between the axes have the form $v \cdot w /|v||w|$ where $v$ and $w$ are chosen from these vectors. These cosines are enumerated in Fig. 1. The angles that correspond to them are those shown in Fig. 2, together with their supplements.

Table 3 gives the components of the Dehn invariants for the non-snub Archimedean polyhedra of edge lengths 1 . For instance the dihedral angles of the truncated tetrahedron are $\pi-2\langle 3\rangle_{2}$ at 6 edges and $2\langle 3\rangle_{2}$ at the remaining 12 , so that its Dehn invariant is

$$
6 V\left[\pi-2\langle 3\rangle_{2}\right]+12 V\left[2\langle 3\rangle_{2}\right]=12 V\left[\langle 3\rangle_{2}\right]
$$

since $V[\pi]=0$. We abbreviate $V\left[\langle p\rangle_{d}\right]$ to $\langle p\rangle_{d}$.

We note that the Dehn invariants of the icosahedron, dodecahedron, and icosidodecahedron with unit edge lengths, namely $60\langle 3\rangle_{5},-30\langle 5\rangle_{1}$, and $30\langle 5\rangle_{1}-60\langle 3\rangle_{5}$, respectively, have zero sum, so Sydler's theorem shows that it is possible to dissect them into finitely many pieces that can be reassembled to form a large cube. This might make an intriguing wooden puzzle if an explicit dissection could be found. (We have no idea how to do this.) 


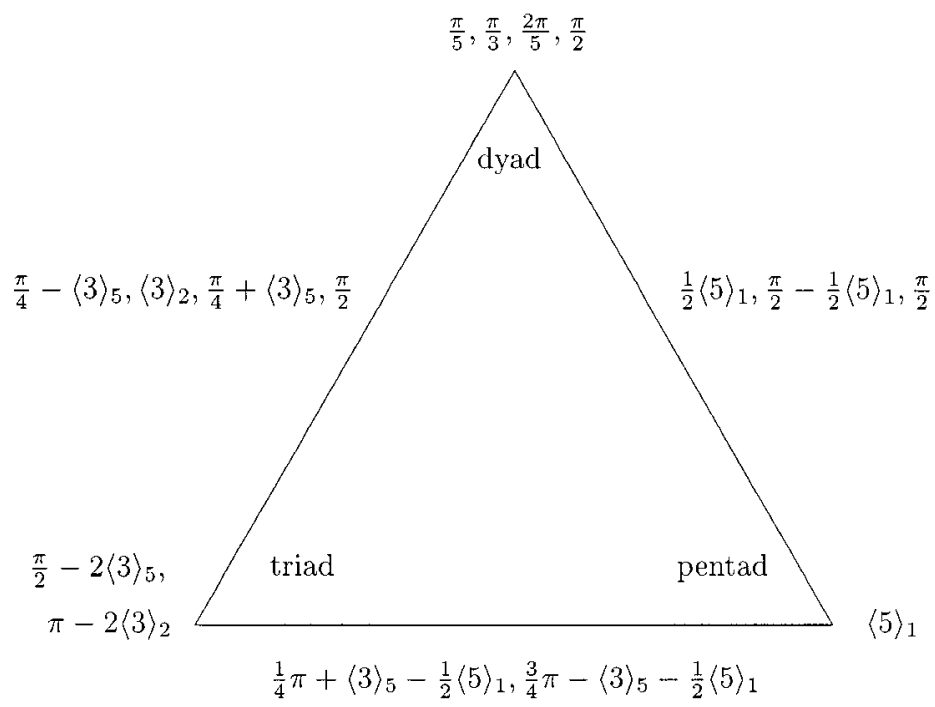

Fig. 2. Angles between axes of fixed rotational symmetry (shown at corners), and between axes of different rotational symmetry (shown at edges).

Table 3. The Dehn invariant for the non-snub unit edge Archimedean polyhedra.

\begin{tabular}{ll}
\hline Tetrahedron & $-12\langle 3\rangle_{2}$ \\
Truncated tetrahedron & $12\langle 3\rangle_{2}$ \\
Cube & 0 \\
Truncated cube & $-24\langle 3\rangle_{2}$ \\
Octahedron & $24\langle 3\rangle_{2}$ \\
Truncated octahedron & 0 \\
Rhombicuboctahedron & $-24\langle 3\rangle_{2}$ \\
Cuboctahedron & $-24\langle 3\rangle_{2}$ \\
Truncated cuboctahedron & 0 \\
Icosahedron & $60\langle 3\rangle_{5}$ \\
Truncated icosahedron & $30\langle 5\rangle_{1}$ \\
& $-30\langle 5\rangle_{1}$ \\
Dodecahedron & $-60\langle 3\rangle_{5}$ \\
Truncated dodecahedron & $60\langle 3\rangle_{5}-30\langle 5\rangle_{1}$ \\
Rhombicosidodecahedron & $-60\langle 3\rangle_{5}+30\langle 5\rangle_{1}$ \\
Icosidodecahedron & 0 \\
Truncated icosidodecahedron & \\
\hline
\end{tabular}




\section{Angles with Algebraic Trigonometric Functions}

It is natural to consider a generalization of our theory that gives a basis for the rational vector space generated by all the angles whose six trigonometric functions are algebraic. What is missing here is the analogue of our Splitting Theorem. If such an analogue were found, the ideal theory would probably go through quite easily.

We ask a precise question: Does there exist an algorithm that finds all the rational linear relations between a finite number of such angles? The nicest answer would be one giving an explicit basis, analogous to our $\langle p\rangle_{d}$.

\section{References}

[Con] J. H. Conway and R. K. Guy, The Book of Numbers, Copernicus, New York, 1996.

[Deh] M. Dehn, Uber den Rauminhalt, Göttingen Nachr. Math. Phys. (1900), 345-354; Math. Ann., 55 (1902), $465-478$.

[Pol] H. Pollard and H. Diamond, The Theory of Algebraic Numbers, second edition, Carus Mathematical Monographs, 9, Mathematical Association of America, Washington, DC, 1975.

[Sah] C.-H. Sah, Hilbert's Third Problem: Scissors Congruence, Pitman, San Francisco, CA, 1979.

[Syd] J. P. Sydler, Conditions nécessaires et suffisantes pour l'équivalence des polyèdres l'espace euclidien à trois dimensions, Comment. Math. Helv., 40 (1965), 43-80.

[Stø] C. Størmer, Sur l'application de la théorie des nombres entiers complexes à la solution en nombres rationnels $x_{1}, x_{2} \ldots x_{n} c_{1} c_{2} \ldots c_{n}, k$ de l'équation $c_{1} \operatorname{arctg} x_{1}+c_{2} \operatorname{arctg} x_{2}+\cdots c_{n} \operatorname{arctg} x_{n}=k \frac{\pi}{4}$, Arch. Math. Naturvid., 19 (1896).

Received April 7, 1998, and in revised form September 2, 1998. 